\title{
Doctor Chatbot - Smart Health Prediction
}

\author{
Seema J, Suman S, Chirag S R, Vinay G, Balakrishna D \\ Department of Information Science and Engineering, Nagarjuna College of Engineering and Technology \\ (Affiliated to VTU), Bengaluru, Karnataka, India
}

\section{ABSTRACT}

Article Info

Volume 8, Issue 3

Page Number : 751-756

\section{Publication Issue}

May-June-2021

\section{Article History}

Accepted : 12 June 2021

Published : 20 June 2021
As in today's world, the number of patients on usual is increasing apace with the amendment in life-style. Queues in hospitals and native doctor's residences are rapidly Increasing. Patients with hectic schedules must spend a significant amount of time waiting to meet the doctor. It takes a long time to heal certain diseases. Heart disease could be a quite common problem worldwide. Many people, both young and old, suffer and die from heart attacks every day. Chatbots will perform tasks such as reducing agent transfers, resolving issues more quickly, improving self-service, and so on. They need constant support to discuss their issues with and to provide them with factual data. This paper introduces a possible solution to provide them with what they're seeking for a chatbot. The projected chatbot would be a heart disease Predictor which is designed for individuals managing any kind of symptoms that connect to the heart. The bot is trained by data collected from numerous Heart Disease-related forums that have a good and wide range of knowledge regarding the heart.

Keywords : Medical Chatbot, Healthcare, Heart Disease Prediction, Data Mining, Support Vector Machine (SVM), Dialogflow.

\section{INTRODUCTION}

A chatbot is a piece of artificial intelligence (AI) software that is capable of simulating oral communication (or a conversation) via electronic messaging applications, blogs, mobile apps, Websites, or the telephone, with a handler in an accepted language. This Technology is obtaining additional widespread principally within the business field and might cut back workforce with such automation. Some employ natural language processing techniques, while others simply search the input for keywords and then return an answer containing the most appropriate keywords/patterns from the data. Cardiovascular disease could be a quite common problem worldwide. However, some areas have a scarcity of physicians. Several of them have fair consultation fees that certain low- income people cannot afford. As a consequence, there is a need for a tool that can assist in the diagnosis of heart problems without the intervention of a specialist, is cost-free, and convenient to use for both adults and adolescents. 
The majority of medical chatbots available are for general use, not unique to a specific medical domain such as cardiology (heart), gastroenterology, or others. The majority of those chatbots cannot schedule appointments with concerned physicians. The aim is to build a conversational system that practices Dialogflow as the front end and the SVM algorithm to identify the dataset and make a prediction whether or not the user is suffering from a heart condition or not. This method will be able to obtain medical records, interpret them, and determine whether or not the patient is suffering from cardiovascular disease. It will also assist individuals in receiving an early diagnosis. Patients would be able to schedule appointments with concerned doctors through this method. Hospitals will be able to provide online diagnostic assistance 24 hours a day, seven days a week with the help of this healthcare bot system.

\section{RELATED LITERATURE}

The literature review aimed to verify and collect data from previous studies and surveys. Some papers were categorized grounded on tools, algorithms, software, and datasets they used at the side of the platform.

Natural Language Processing, Framework for Emotion Recognition at the side of spatial-Temporal Context Analysis, Multi-modal Approach was employed for outlining an Emotion Recognition Model to categorize, collection of the training data for emotion Recognition, and logical thinking, Continuous Emotional observation exploitation information in the form of text, image, video, audio format [1]. SWITCH is made up of two main components: a web-based dashboard and an android app that uses Health Intervention Tools and builds a custom medical algorithm for Android conversations under the LINE weight management channel and a Health Promotion- level power balance [2]. The chatbot was developed for use by cancer patients anyone who wishes to understand more about cancer.
The user message is sent via WhatsApp, Facebook, and other social media platforms. Chatbot uses the Python library to retrieve data through cancer forums and archive it in a native database, and processes data in advance using the NLTK collectively accessible in Python. the communicator takes the downloaded information and produces a personal result [3]. VDMS is a web-based chatbot that was constructed by exploiting an open-source PHP interpreter designed for AIML which is known as program-o. The native cognitive content of VDMS on polygenic disease is formed by the exploitation of AIML pattern tags. In addition, a pattern matching algorithm was used in this. A test was performed where a group consisting of ten participants asked inquiries to VDMS from that the chatbot might answer up to sixty-fifth of queries that the users were glad about [4] Farm bot is a robot agriculture platform that uses natural language processing to understand user queries in their native tongue. This bot was created using a gradient descent algorithm and a training database with a built-in network database. To generate voice- generated responses for the user, this chatbot used the Web Speech API interface. The chatbot also has a prediction algorithmic rule for predicting the lengthy price of agricultural goods, which had a maximum squared proportion error of 0.1904 percent for the data used for prediction when checked. [number five] Conversation created for the usage of Eclipse applications on Windows software that uses of Java programming language. Using java applets to build a discussion panel the website for this chatbot provides a multi list to modify content. This chatbot was designed with a simple mindset in mind, and it only answers questions found in the database [6]. The MediAssistantEdge platform made use of MySQL, AIML, swftools, Red5, RTMP, HTTP, and the SingleSupply Biasing Process. The advanced framework included two separate, namely the DocBot system, which streamlined a diagnostic process and increased patient-physician coordination [7]. MedBot makes use of datasets from DoctorMe Apps, Dialogflow API, 
Natural Language Understanding (NLU), and Pattern matching algorithm. Chatbots are often conveniently deployed in web chat systems like Facebook, Hangouts, or Line using the appropriate APIs. The test performance determines the proposed system's potential (results) [8]. The Decision Tree Algorithm as well as the Cleveland Clinic Foundation (Cleveland Data) were analysed using the WEKA and KEEL tools for prediction of heart disease, resulting in 87.41722 percent positively distinguished instances. ECR, cholesterol, discomfort, rapid blood sugar, and quantity are some of the main parameters used. Obtaining a pulse, etc. To fill in missing values in the database, the A11possible- MV Algorithm was used [9]. Machine learning and ai algorithms were contrasted. The MAS (Multi-Agent System) help tool kit is included inside the conversation manager to explain predicted behaviour and RL (Strengthening Learning) to make the learning simpler. The bot was trained using RL [10] in datasets from the site. SVM, k-NN, Nave Bayes, and Porter Stemming Algorithms are being used in combination with AIML, Java \& Pascal, MySQL, NLP, and some Heart Diseases datasets for construct a heart disease prediction system [11]. Cardiovascular diagnostic measures have been reduced using the feature-subset collection technique, which removes appropriate data from a database when removing inactive data. The genetic algorithm reduces the number of traits in a data collection by using genetic search, rendering it simpler to search. Using a compilation of record 909 data sets with 13 symbols [12]. Pharmabot is a standalone medical chatbot built with Visual C\# on front end or Ms Access mostly on back end. The Left-Right Parsing Algorithm would be used to test the user's data. The chatbot's user-friendliness, acceptability, precision, and speed were evaluated by a community of pharmacy students and paediatricians [13].

\section{PROPOSED METHOD}

According to the literature review in the preceding portion, the following techniques and technology may be used to improve chatbot architecture, research, and accuracy when building a chatbot or communicative device for any domain:

\section{A. Tools and Technologies}

Datasets: Many free databases are accessible on different domains on platforms such as Kaggle, GitHub, and others. It's also open on some websites directly. There are three databases used for the detection of heart failure, for example. Data were obtained at three locations: the Cleveland Clinic Foundation, the Hungarian Institute of Cardiology, University Hospital in Switzerland, and the Cleveland Clinic Foundation.

ML/DM Algorithms: Following data processing, algorithms including the support vector machine (SVM) may be used to conduct classification and regression analysis. Each of the classes are assigned their own training directory. The SVM training algorithm is indeed a binary line divider that creates a model that categorizes new models into two groups. It has a higher degree of precision than other algorithms.

Dialogflow (API.ai): It is a free Google Technology that supports AI to provide users with better ways to connect with the product or service using voice and text-based contact methods (using NLP). Dialogflow uses Google's machine learning platform and services including Google Cloud Speech-to-Text.

MATLAB: Other languages are used to create computer IDEs that allow for matrix manipulation, data editing, program execution, UI design, and advanced integration systems.

WEKA 3: Weka is really an open data analysis platform / programme that uses a collection of applied machine learning algorithms to process data. It has resources for combining, rewinding, pre-processing results, merging, segmentation, and visualisation that can be used collaboratively. 
AIML: Artificial Intelligence Mark-up Language (AIML) is an XML language for creating chat agents. Some chatbots can react to user messages using the AIML process, which

may result in constructive and significant responses. It's simple to edit, learn, and use, and it's also really secure.

Ngrok: Ngrok is a network tunneling application for checking APIs and webhooks on a local server. There are two different versions of the game to choose from. We're using ngrok.io's free version, which requires registration. It is used here to link Dialogflow to the backend operation (SVM) using a webhook and the flask library in Python.

\section{SYSTEM DESIGN}

The process of defining system components such as modules, structure, components, and their integration and system data based on defined needs is known as system design. Designs can be illustrated using graphical or textual models.

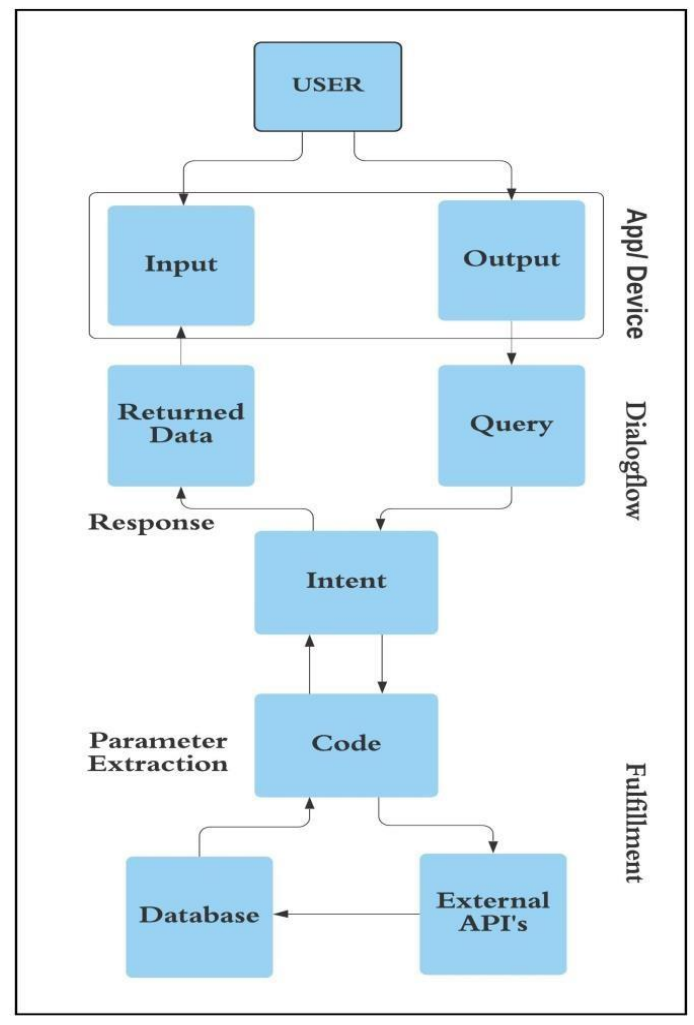

Fig. 1. System Working Model
Input Expression/Query: The User inputs the data to the System. the data includes entries of a number of the attributes of the center dataset like an electrocardiogram, blood pressure, etc.

Intent Matching: for every agent, you outline several intents, where your combined intents will handle a whole speech. Dialogflow matches the end-user expression to the intent of your agent.

Webhook request: a Webhook may be an approach for an app to supply different applications with realtime info. you

furthermore may have to be compelled to set up a URL in your app that's accessible from the general public web. Ngrok may be a server connected with the python program and is employed to transfer information dynamically from python to Dialogflow victimization ngrok server link.

Conversational response through Dialogflow: currently, Dialogflow encompasses a less complicated response to administer back, i.e. respondent whether or not the user is plagued by Heart Diseases or not. The chatbot is additionally trained for Booking Appointments with related Doctor.

\section{CONCLUSION}

The most implemented chatbots use AI and Data Mining algorithms such as SVM, Decision tree, Genetic algorithm, Naïve Bayes, Pattern Matching, NLP, and more using tools such as WEKA or MATLAB for analysis or execution. Some algorithms were tested and compared based on accuracy after giving input datasets. Some chatbots used AIML for the QA structure. This paper specifies Dialogflow as the Conversation Interface. Under the medical domain, the data mining model which gives the best accuracy for predicting these diseases from the heart 
disease dataset was the Support Vector Machine algorithm. AIML can also be used for creating chatbots by using XML format to store questions and answers. MATLAB and Weka tools were used for analysis purposes including pre-processing, clustering, and graph plotting.

\section{REFERENCES}

[1]. Kyo-Joong Oh, DongKun Lee, ByungSoo Ko, Ho-Jin Choi; "A Chatbot for Psychiatric Counseling in Mental Healthcare Service Based on Emotional Dialogue Analysis and Sentence Generation";2375-0324/17 \$31.00 @2017 IEEE, 2017 IEEE 18th International Conference on Mobile Data Management.

[2]. Chin-Yuan Huang, Ming-Chin Yang, Chin-Yu Huang, Yu - Jui Chen, Meng-Lin Wu, Kai-Wen Chen; "A Chatbot- supported Smart Wireless Interactive Healthcare System for Weight Control and Health Promotion"; 978-1-53866786-6/18/\$31.00 @2018 IEEE, Proceedings Of the 2018 IEEE IEEM.

[3]. Belfin R V, Ashly Ann Mathew, Blessy Babu, Shobana A.J., Megha Manilal; "A Graph Based Chatbot for Cancer Patients"; 978-1-53869533-3/19/\$31.00 C2019 IEEE, 2019 5th International Conference on Advanced Computing \& Communication Systems (ICACCS).

[4]. Shafquat Hussain, Prof. Athula Ginige; "Extending a conventional chatbot knowledge base to external knowledge source and introducing user based sessions for diabetes education"; 978-1-5386-53951/18/\$31.00@2018 IEEE, 2018 32nd International Conference on Advanced Information Networking and Applications Workshops.

[5]. Prof. Mrs. L. Kannagi M.E., Ramya .C, Shreya .R, Sowmiya .R; "Virtual Conversational Assistant - "The FARMBOT"; International
Journal of Engineering Technology Science and Research, ISSN 23943386 Volume 5, Issue 3 March 2018.

[6]. M. Dahiya, "A Tool of Conversation: Chatbot"; International Journal Of Computer Sciences and Engineering, Volume-5, Issue-5, E-ISSN: 2347-2693, Published:30/May/2017.

[7]. Amiya Kumar Tripathy, Rebeck Carvalho, Ajit Puthenputhussery, Nikita Chhabhaiya, Bijoy Anthony; "MediAssistEdge - Simplifying Diagnosis procedure \& Improving patient doctor connectivity"; 978- 1-4799-81878/15/\$31.00 (2015 IEEE, 2015 International Conference On Technologies for Sustainable Development (ICTSD- 2015), Feb. 04-06, 2015, Mumbai, India.

[8]. Shafquat Hussain, Prof. Athula Ginige; "Extending a conventional chatbot knowledge base to external knowledge source and introducing user based sessions for diabetes education"; $\quad 978-1-5386-5395-\quad 1 / 18 / \$ 31.00$ (C)2018 IEEE, 2018 32nd International Conference on Advanced Information Networking and Applications Workshops.

[9]. Purushottam, Richa Sharma, Prof. (Dr.) Kanak Saxena; "Efficient Heart Disease Prediction System using Decision Tree"; ISBN:978-14799- 8890-7/15/\$31.00 (C)2015 IEEE, International Conference on Computing, Communication and Automation (ICCCA2015).

[10]. Kevin MUGOYE, Dr. Henry OKOYO, Dr. Sylvester MCOYOWO; "Smart-bot Technology: Conversational Agents Role in Maternal Healthcare Support"; IIMC International Information Management Corporation, 2019 ISBN: 978-1-905824-63-2, IST-Africa 2019 Conference Proceedings.

[11]. Mrs. Rashmi Dharwadkar, Dr. Mrs. Neeta A. Deshpande; "A Medical Chatbot"; International Journal of Computer Trends and Technology (IJCTT)-Volume 60 Issue 1- June 2018. 
[12]. M. ANBARASI, E. ANUPRIYA, N.CH.S.N. IYENGAR; "Enhanced Prediction of Heart

Disease with Feature Subset Selection using Genetic Algorithm"; International Journal of Engineering Science and Technology Vol. 2(10), 2010, 5370-5376.

[13]. Benilda Eleonor V. Comendador, Bien Michael B. Francisco, Jefferson S. Medenilla, Sharleen Mae T. Nacion, and Timothy Bryle

[14]. E. Serac; "Pharmabot: A Pediatric Generic Medicine Consultant Chatbot"; Journal of Automation and Control Engineering Vol. 3, No. 2, April 2015.

\section{Cite this article as :}

Seema J, Suman S, Chirag S R, Vinay G, Balakrishna D, " Doctor Chatbot - Smart Health Prediction", International Journal of Scientific Research in Science and Technology(IJSRST), Print ISSN : 2395-6011, Online ISSN : 2395-602X, Volume 8, Issue 3, pp.751756, May-June-2021. Available at doi : https://doi.org/10.32628/IJSRST2183172 